\title{
Experience in Offloading Protocol Processing to a Programmable NIC
}

\author{
Arthur B. Maccabe, Wenbin Zhu* Jim Otto, Rolf Riesen ${ }^{\dagger}$
}

April 2002

\begin{abstract}
Offloading protocol processing will become an important tool in supporting our efforts to deliver increasing bandwidth to applications. In this paper we describe our experience in offloading protocol processing to a programmable gigabit Ethernet network interface card. For our experiments, we selected a simple RTS/CTS (request to send/clear to send) protocol called RMPP (Reliable Message Passing Protocol). This protocol provides endto-end flow control and full message retransmit in the case of a lost or corrupt packet. By carefully selecting parts of the protocol for offloading, we were able to improve the bandwidth delivered to MPI applications from approximately $280 \mathrm{Mb} / \mathrm{s}$ to approximately $700 \mathrm{Mb} / \mathrm{s}$ using standard, 1500 byte, Ethernet frames. Using "jumbo", 9000 byte, frames the bandwidth improves from approximately $425 \mathrm{Mb} / \mathrm{s}$ to $840 \mathrm{Mb} / \mathrm{s}$. Moreover, we were able to show a significant increase in the availability of the host processor.
\end{abstract}

\section{Introduction}

As network transmission rates have increased, it has become increasingly difficult to deliver this increase to applications. Delivering this bandwidth to applications requires that several bottlenecks, including the speed of the

${ }^{*}$ A. B. Maccabe and W. Zhu are with the Computer Science Department, University of New Mexico, Albuquerque NM 87131-1386. This work was supported in part by Sandia National Laboratories under contract number AP-1739.

${ }^{\dagger}$ J. Otto and R. Riesen are with the Scalable Computing Systems Department, Sandia National Laboratories, Org 9223, MS 1110, Albuquerque, NM 87185-1110
I/O bus, memory copy rates, and processor capacity, be addressed. In this paper, we consider the bottleneck associated with processor capacity. In particular, we consider migrating part of the processing associated with communication protocols from the host processor to a programmable NIC (Network Interface Controller).

This offloading of protocol processing has two significant benefits. First, by removing the processor capacity bottleneck, it results in improved communication bandwidth. Second, by moving protocol processing to the NIC, we will improve the availability of the host processor for use by application programs.

While there are clear advantages associated with offloading protocol processing, we must be careful in how much work we offload to the NIC. Current NICs have fairly severe limits in both memory capacity and processing power. For example, the Alteon Acenics only have $2 \mathrm{MB}$ of local RAM and two $88 \mathrm{MHz}$ processors.

The remainder of this paper is organized as follows. The next section explores the nature of the processor capacity bottleneck. In Section 3 we describe the protocol that provided the basis for our offloading experiments. Section 4 describes the steps that we undertook in offloading parts of this protocol. Section 5 describes the results of our offloading experiment. Section 6 discusses related work. Finally, Section 7 presents our conclusions and plans for further work. 


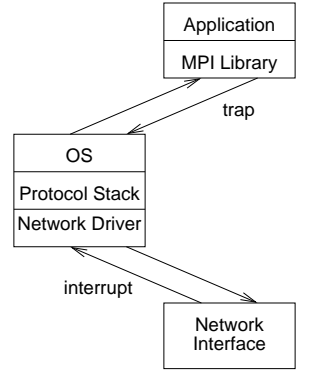

Figure 1: Typical Implementation of a Protocol Stack

\section{Understanding the Processor Ca- pacity Bottleneck}

Given the instruction processing rates of modern processors, it may be hard to imagine that processor capacity could represent a significant bottleneck in any aspect of communication or computation. Moreover, considering the limited instruction rates of the processors available on NICs, it may seem contradictory that offloading any processing to a NIC processor will provide any significant benefit. Ultimately, the issue boils down to the costs associated with interrupts and traps on modern processors.

To support high-performance, parallel computations, we are interested in performance, as measured by MPI (the Message Passing Interface)[8] applications. Figure 1 shows the typical implementation of a communication protocol stack. Because the host processor is involved in every packet transmission, the strategy shown in Figure 1 implies a significant amount of overhead. In particular, the host processor will receive a large number of interrupts to handle incoming packets.

If not carefully controlled, communication overhead can quickly dominate all other concerns. As an example we consider minimum inter-arrival times for 1500 byte Ethernet frames (ignoring the preamble, frame start delimiter, Ethernet header, checksum, and inter-frame gap.) Table 1 summarizes frame inter-arrival times for several network transmission rates. As an example, the interarrival time for $100 \mathrm{Mb}$ Ethernet was calculated as:

$$
1500 \mathrm{~B} \times \frac{8 \mathrm{~b}}{\mathrm{~B}} \times \frac{1}{100 \mathrm{Mb} / \mathrm{s}}=120 \mu \mathrm{s}
$$

Through indirect measurement, we have observed that
Table 1: Minimum Inter-arrival Time for 1500 Byte Ethernet Frames

\begin{tabular}{l|l} 
Network Rate & Inter-arrival Time \\
\hline $10 \mathrm{Mb} / \mathrm{s}$ & $1200 \mu \mathrm{s}$ \\
$100 \mathrm{Mb} / \mathrm{s}$ & $120 \mu \mathrm{s}$ \\
$1 \mathrm{~Gb} / \mathrm{s}$ & $12 \mu \mathrm{s}$ \\
$10 \mathrm{~Gb} / \mathrm{s}$ & $1.2 \mu \mathrm{s}$
\end{tabular}

interrupt overhead for an empty interrupt handler is between five and ten microseconds on current generation computing systems. Interrupt overhead measures the processing time taken from the application to handle an interrupt. Interrupt latency, in contrast, measures how quickly the computing system can respond to an interrupt. By throwing away all work in progress on a pipelined processor, a CPU designer could reduce the latency of an interrupt; however, the interrupt overhead would not be reduced as the work that was discarded will need to be reproduced when the application is re-started after the interrupt has been processed.

Assuming that interrupt overheads are 10 microseconds, including packet processing, we see that the communication overhead will be approximately $83 \%$ (10 out of every 12 microseconds is dedicated to processing packets) for Gigabit Ethernet!

Two approaches are commonly used to reduce the communication overhead for Gigabit Ethernet: jumbo frames and interrupt coalescing. Jumbo frames increase the frame size from 1500 bytes to 9000 bytes. In the case of Gigabit Ethernet, this increases the minimum frame inter-arrival time from 12 microseconds to 72 microseconds. This will reduce the communication overhead substantially. Unfortunately, this only works for larger messages and will not be particularly helpful for 10 Gigabit Ethernet.

Interrupt coalescing holds interrupts (at the NIC) until a specified number of packets have arrived or a specified period of time has elapsed, which ever comes first. This reduces communication overhead by throttling interrupts associated with communication. When a constant stream of frames is arriving at a node, interrupt coalescing amortizes the overhead associated with an interrupt over a collection of frames. This approach will work well, with 
higher speed networks, but introduces a high degree of variability in the latency for small messages.

Our approach to dealing with this problem is to offload small parts of the protocol processing onto the NIC. In particular, we offload message fragmentation and reassembly. In addition, we avoid unnecessary memory copies which further reduces processor overhead.

\section{The RMPP Protocol}

For our experimentation, we chose a simple RTS/CTS protocol called RMPP[10]. RMPP supports end-to-end flow control and message-level retransmission in the event of a dropped or corrupt data packet. Figure 2 presents a graphical illustration of the packets used in this protocol. Because it is not important in the context of this paper, we will not discuss the fault tolerance aspects of this protocol. Instead, we will focus on the flow control properties.

The first step in sending a message is the transmission of an RTS (Request To Send) packet from the sender to the destination (receiver). This packet includes an RMPP header (indicating that it is an RTS packet) and the initial data of the message. The packet will be filled with message data up to the MTU (Maximum Transmission Unit) of the underlying network. Importantly, the RTS packet should include headers for all upper level protocols. For example, a header constructed by the MPI library.

When the destination receives an RTS packet, it can use the data in the RTS packet (i.e., the headers for the upper level protocols) to determine where the message should be delivered. For example, it could match the incoming message to a pre-posted MPI receive issued by an application on the receiving node. Once the destination of the incoming message is known, the receiver can reply with a CTS (Clear To Send) packet. This packet indicates the number of data packets that the sender is permitted to send before receiving the next CTS.

Each CTS enables the transmission of $n$ data packets and is used to control the flow of data from the sender. The actual value of $n$ is at least 1 and will reflect the minimum of: the space required for 16 data packets, the space available in the application (this may reflect "pinned" pages), and the buffer space available on the NIC.

When the sender receives a CTS packet, it transmits the allowed number of data packets and, assuming that the message contains more data, sends an $\mathrm{RSM}^{1}$ (Request to Send More) to request the transmission of more data packets. Once the sender has transmitted all of the data packets, it sends an END packet, indicating that it is done sending the current message. The receiver acknowledges receipt of the message by sending an ACK packet. Upon receiving the ACK packet, the sender can reclaim any state related to the message transmission (the delivery is complete) and send a CLEANUP packet to the receiver. Finally, when it receives a CLEANUP packet, the receiver knows that the sender knows that the message has been delivered and the receiver can reclaim any resources associated with the message transmission. (If the CLEANUP packet is dropped, the receiver will time out on receiving the CLEANUP and will then reclaim these resources.)

In considering the RMPP protocol that we have described, notice that all management decisions are made, by the receiver, when handling an RTS or RSM packet. By the time the receiver has generated the CTS packet, it has made all of the significant management decisions, including: where the cleared data packets will be placed (in the application's space) and the NIC buffer space that will be used for incoming packets. Once these decisions have been made, responses to all other packets (excluding the END, ACK, and CLEANUP) packets are straightforward. This observation lead to our approach for protocol offloading.

\section{Steps in Protocol Offloading}

The RMPP protocol described in the previous section was developed as a reliable transport layer for implementing the Portals 3.0 API[1] on Myrinet. Our first step, step 0, was to make the protocol run on Gigabit Ethernet using the Alteon ACENICs. The next two steps involved offloading the handling of data packets: first on the receiver, then on the sender. Finally, we improved the performance of the protocol by moving the RTS packets earlier in the stream of data packets to maintain a constant stream of data packets.

\footnotetext{
${ }^{1}$ Like the initial RTS, each RSM is actually a flag in the header of the last data packet. For this presentation, it is easier to think of this as a separate packet and the actual implementation as an optimization.
} 


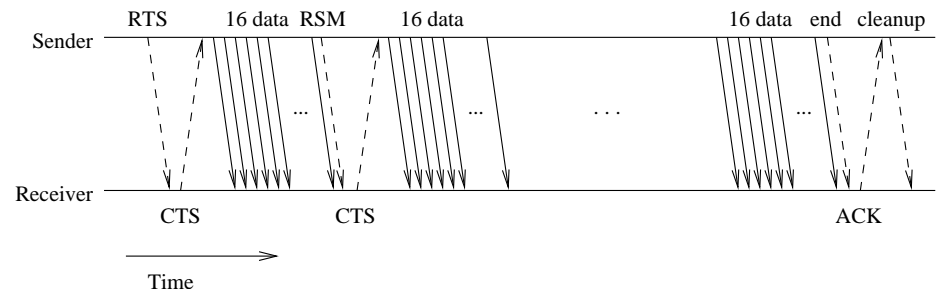

Figure 2: Message exchanges in the RMPP protocol

\subsection{Step 0: Porting RMPP to Gigabit Eth- ernet}

Implementation of the RMPP protocol is based on two Linux kernel modules, the Portals module and the RMPP module, and a Myrinet control program (MCP), the packet MCP. The primary responsibility of the packet MCP is to relay packets between the RMPP module and the Myrinet network. In addition, this control program coalesces interrupts and provides buffers for incoming and outgoing packets. The RMPP module is responsible for scheduling the use of the resources provided by the NIC (buffers) and implementing the RMPP protocol. The Portals module implements the Portals API. From the perspective of the RMPP module, the Portals module provides the final destination for each incoming message when the initial RTS for a message is received.

The decomposition of this implementation into the Portals module, the RMPP module, and the packet MCP reflects a partitioning based on policy versus mechanism. The Portals module provides the policy for message delivery while the RMPP module provides the mechanism needed to implement this policy. Similarly, the RMPP module provides the policy needed to control multiple flows, while the packet MCP implements these flows by sending and receiving network packets.

Before offloading parts of the RMPP protocol onto the Alteon ACENIC Gigabit Ethernet NIC, we decided to integrate this driver into the Linux Network stack. In particular, we established an skbuf wrapper for the packets used by the RMPP module. Linux uses skbufs as the basis for all network traffic. By providing a translation between skbufs and RMPP packets, this wrapper makes it easy to use the RMPP protocol with any of the existing Linux network device drivers, including the driver for the

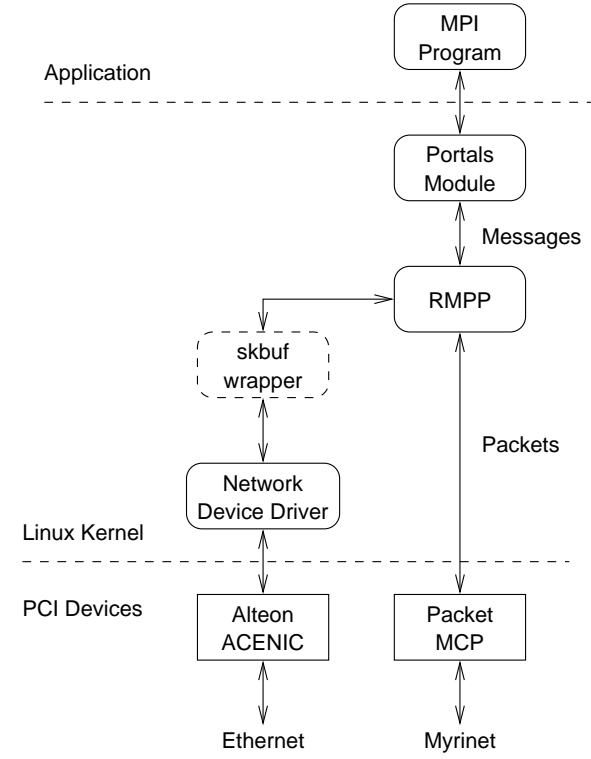

Figure 3: The Structure of our Initial RMPP Implementation

Alteon ACENIC. Figure 3 illustrates the structure of our initial implementation, including the skbuf wrapper.

\subsection{Step 1: Receive Offloading}

Once we had our initial implementation working, we offloaded the processing of incoming data packets from the RMPP module to the NIC control program. Whenever the RMPP module generates a CTS packet, it also pushes physical addresses for the memory region in which the "cleared" packets will be placed. Now, when the cleared data packets arrive at the NIC, they can be transferred directly to application memory. The resulting information 


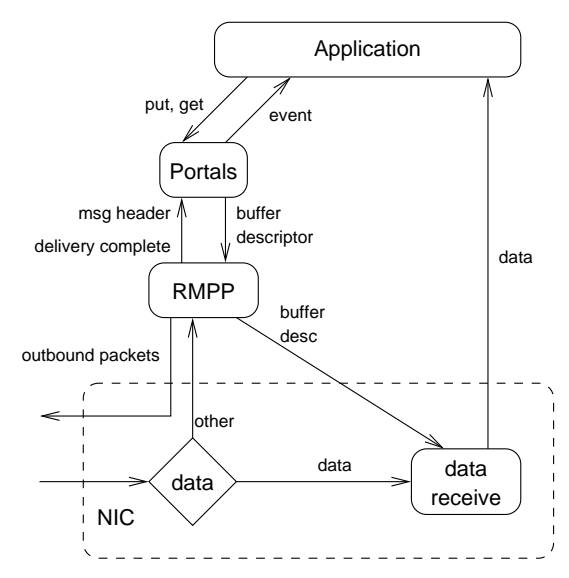

Figure 4: Processing Incoming Data Packets on the NIC

and packet flow is presented in Figure 4.

There are two significant advantages that should be apparent from this modification. First, we have significantly reduced the number of interrupts that the host processor will need to field. Second, we have reduced the number of copies for incoming data packets - these packets are delivered directly to the application with no intermediate copies.

Moreover, we have not significantly increased the amount of work that the NIC needs to do. As before, the NIC needs to schedule a DMA operation to transfer the data packet from NIC memory to host memory. In the previous implementation the target address was determined by the next entry in a queue of memory descriptors provided by the kernel. Now, the NIC control program needs to use information in the incoming packet header as an index into an array of physical addresses to determine the host memory target address for this DMA operation. In essence, the NIC needs to be able to demultiplex multiple incoming data streams. In addition, the NIC needs to watch for dropped packets and notify the RMPP module whenever it detects an out of order packet. (The RMPP module uses a timeout to detect loss of the last packet in a group of cleared packets.) Neither of these activities should have a significant impact on the responsiveness of the NIC.

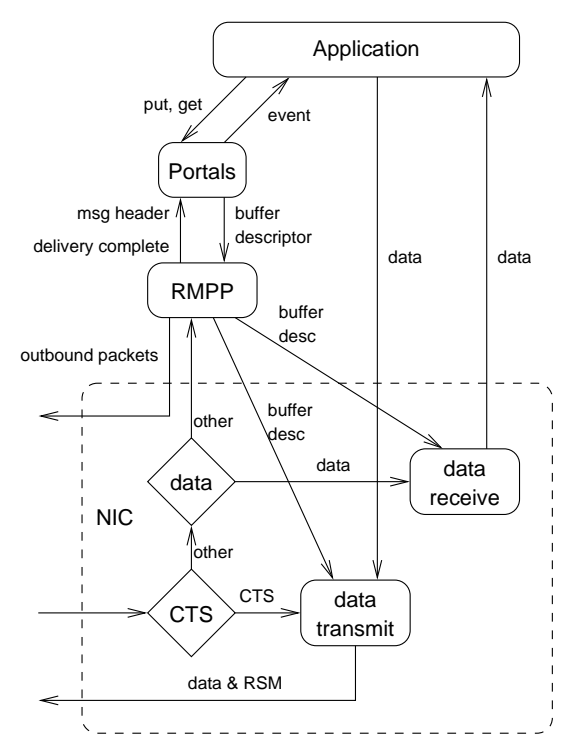

Figure 5: Sending from the NIC

\subsection{Step 2: Send Offloading}

In the next step we offloaded the processing associated with sending messages from the RMPP module to the NIC control program. With this modification, when an application program initiates a message send, the RMPP module sends the initial RTS packet and pushes a descriptor of the outgoing message buffer to the NIC. This descriptor includes the physical addresses needed to transfer parts of the message from host memory to NIC memory during the formation of the data packets. Given this information, the NIC control program is now in a position to respond to incoming CTS packets by building and sending the data packets that are cleared by the CTS. Moreover, the NIC control program can also generate RSM packets. Figure 5 illustrates the information and packet flow that results from this modification.

The primary advantage in this step is avoiding the memory copy from the application space to the intermediate kernel buffers. In addition, we save the occasional interrupt that would be needed to process incoming CTS packets. 


\subsection{Step 3: Pipeline Management}

In all of the earlier implementations, the RSM is sent with the last of the cleared data packets. This creates a bubble in the stream of data packets while the receiver processes the RSM, generates the CTS and sends it back to the sender. In the final step, we experimented with moving the RSM earlier in the stream to avoid these bubbles.

\section{Results}

We evaluate our modifications by measuring improvements to bandwidth and processor availability. All of the results reported in this section we obtained using two systems connected by a "crossover" Gigabit Ethernet cable. Each node has a $500 \mathrm{MHz}$ Pentium III.

\subsection{Bandwidth Results}

We measure bandwidth using a traditional ping-pong test, using MPI for all communication. Pseudocode for this measurement is shown in Figure 6. In this test, the ping process sends a message to a pong process. The pong process replies by returning the original message. The test is repeated for a collection of message sizes and within each message size, several times to account for memory effects. For each message size, the test reports the latency. Bandwidth is calculated by dividing the message size by the latency. The actual measurement that we use reports the minimum, maximum, and average latency.

Others measure availability during communication by simply flooding a node with messages. By using a pingpong test, we can easily measure the overhead associated with sending and receiving messages. In order to observe full bandwidth, our ping-pong test starts by sending several messages. This eliminates the bubble that would otherwise occur while the message is turned around by the application process.

Figure 7 presents bandwidth curves for the original implementation, an implementation that offloads receive handling, and an implementation that offloads both send and receive handling. As can be seen, each modification to the implementation results in a significant improvement in the observed bandwidth. Offloading the processing associated with incoming data packets results in a band-

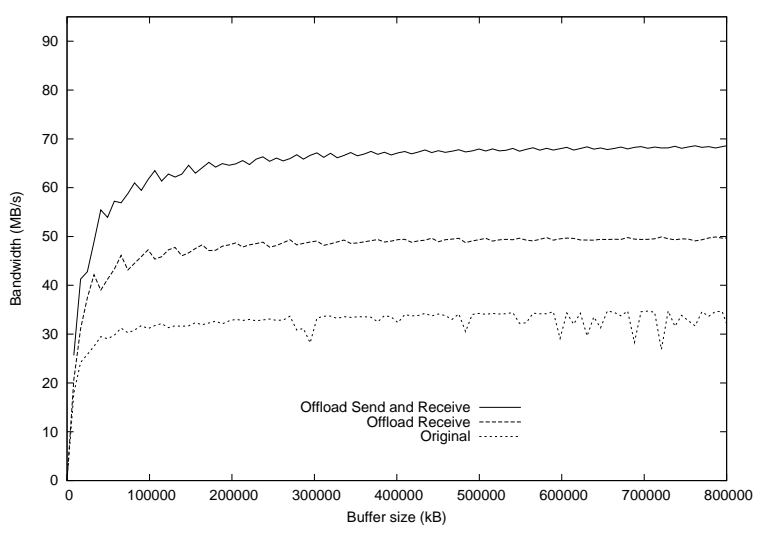

Figure 7: Bandwidth Improvement for Standard (1500 Byte) Frames

width increase from $35 \mathrm{MB} / \mathrm{s}$ to $50 \mathrm{MB} / \mathrm{s}$, a $30 \%$ improvement. Additionally, offloading the processing associated with sending messages increases the bandwidth to $69 \mathrm{MB} / \mathrm{s}$, an addition 28\% improvement.

To gain insight into the improvement observed in Figure 7, we calculated the per packet latency. For the original implementation this value stabilizes at approximately 40 microseconds. When we offload receive processing, this values stabilizes at approximately 27.5 microseconds, an improvement of 12.5 microseconds per packet. When we also offload the send processing, this value stabilizes at approximately 20 microseconds, an additional savings of 7.5 microseconds per packet.

Because the only significant improvement associated with offloading send processing is the avoidance of a memory copy, we conclude that the cost of copying a packet from application space to kernel space is close to 7.5 microseconds. Moreover, we conclude that the additional savings ( 5 microseconds) observed in offloading receive processing is due to the elimination of the per packet interrupt. That is, the elimination of the host processor bottleneck.

After offloading the processing associated with sending messages and receiving data packets, we measured the performance improvements when moving the RSM earlier in the stream. Figure 8 presents the bandwidth improvements associated with moving the RSM earlier in the stream of data packets. The "Pre 0" reflects the de- 
Table 2: Activities in the Bubble and their Approximate Times

\begin{tabular}{l|r} 
Step & $\mu \mathbf{s}$ \\
\hline Transmit data packet with RSM & 15 \\
Receive packet and recognize RSM & 5 \\
Transfer data to application memory & 15 \\
Transfer header to OS memory & 5 \\
Interrupt host processor & 10 \\
Process header in and generate CTS & 5 \\
Transfer addrs for cleared pkts to NIC & 5 \\
Transfer CTS to the NIC & 5 \\
Transmit CTS & 5 \\
Processing CTS & 5 \\
\hline Total & 75
\end{tabular}

communication are run in two separate processes.

Netperf was developed to measure the performance of TCP/IP and UDP/IP. It works very well in this environment. However, there are two problems with the netperf approach when applied to MPI programs. First, MPI environments typically assume that there will be a single process running on a node. As such, we should measure processor availability for a single MPI task while communication is progressing in the background (using non-blocking sends and receives). Second, and perhaps more important, the netperf approach assumes that the process driving the communication relinquishes the processor when it waits for an incoming message. In the case of netperf, this is accomplished using a select call. Unfortunately, many MPI implementations use OS-bypass. In these implementations, waiting is typically implemented using busy waiting. (This is reasonable, given the previous assumption that there is only one process running on the node.)

To avoid these problems, we modify the body of the outer loop for the ping process used to measure bandwidth. The new loop body is presented in Figure 9. On each iteration of the ping process posts an nonblocking send and a nonblocking receive. It then enters a loop in which it polls for completion of the receive. Inside of this loop, the ping process simulates work by delaying for an interval specified by the polling interval, $p$. Notice that the total amount of work to be done drives the measure-

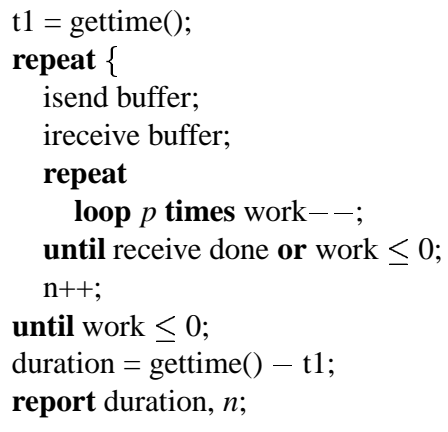

Figure 9: Measuring Processor Availability

ment. To determine processor availability, we compare the time it takes to complete this code when the pong process sends reply messages versus the time when the pong process simply consumes the first message.

Figure 10 illustrates the relationships between polling interval, observed bandwidth, and observed processor availability when using messages with $32 \mathrm{~KB}$ of data. In examining the graphs in this figure, we first note that when we offload both send and receive the bandwidth and processor availability are both relatively high, $42 \mathrm{MB} / \mathrm{s}$ and $85 \%$, respectively. When we only offload the receive processing, the bandwidth is significantly higher than it is for the original implementation $(38 \mathrm{MB} / \mathrm{s}$ versus $21 \mathrm{MB} / \mathrm{s})$, but the host processor availability is the same $(60 \%)$. In essence, we are using the same amount of processing to handle more communication.

Given the tradeoff between processor availability and bandwidth, it is often difficult to easily characterize performance improvements. One approach is to fix the bandwidth and compare the corresponding availability is. As an example, if we fix the bandwidth at $21 \mathrm{MB} / \mathrm{s}$, the processor availability is approximately $60 \%, 80 \%$, and $95 \%$, for the original implementation, receive offload, and receive and transmit offload, respectively. An improvement of $33 \%$ for offloading receive processing and an improvement of $58 \%$ for offloading both send and receive processing.

It is also helpful to consider the effective bandwidth, the product of the availability and the bandwidth. Figure 11 presents effective bandwidth graphs when the message size is $32 \mathrm{~K}$. Here, the improvement becomes much more 


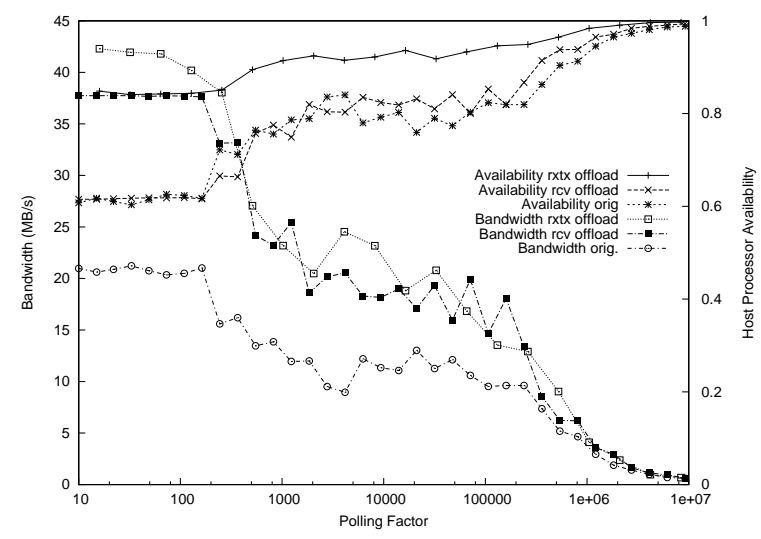

Figure 10: Communication Bandwidth and Processor Availability (Message Size is $32 \mathrm{~K}$ )

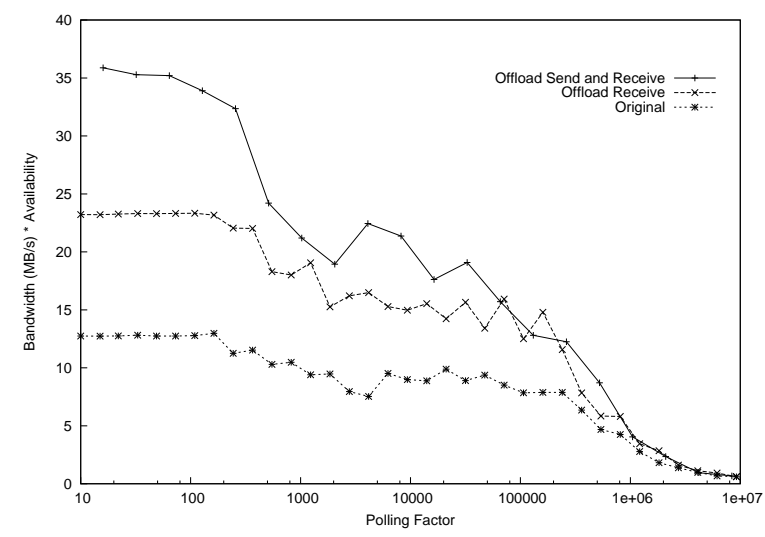

Figure 11: Effective Bandwidth (Message Size is 32K)

apparent. When we offload receive processing, the effective bandwidth improves from $13 \mathrm{MB} / \mathrm{s}$ to $23 \mathrm{MB} / \mathrm{s}$, a factor of 1.77. When we offload both send and receive processing, the effective bandwidth goes to $36 \mathrm{MB} / \mathrm{s}$, a factor of 2.77 improvement.

\section{Related Work}

Earlier work in the Scalable Systems Lab at UNM [3, 13] showed that offloading fragmentation and defragmentation for IP (Internet Protocol) packets could result in a significant improvement in communication bandwidth and host processor availability. Like the work reported in this paper, IP fragmentation studies were based on the Alteon Acenic. While the IP fragmentation work was aimed at improving the performance of IP, the work reported in this paper aims to improve the performance of MPI communication.

To avoid the problems associated with memory copies, communication latency, and communication overhead, several groups have proposed protocols that support "OS bypass" $[2,12,6,9]$. In concept, each process gets its own virtual network interface which it can use directly (bypassing the need to go through the operating system). Needless to say, these proposals do not entirely bypass the OS. They rely on interaction with the OS to enforce resource protection (usually limited to address translation) or to ensure that resources (page frames) are available. In the OS bypass strategy, memory copies are avoided by using buffers in the application memory rather than buffers in the operating system (as such, the application is in control of when copies need to be made). Communication latency is reduced by avoiding the need to trap into OS for sends and interrupt the OS for receives. Communication overhead is also reduced by the elimination of interrupts.

While we borrow many ideas from OS bypass, we use the OS to control the use of network resources and to match incoming messages with their final destination. When MPI applications try to overlap communication with computation, having a mechanism to match incoming messages with pre-posted receives offers significant performance advantages [7]. Providing this mechanism requires some intervention by the OS, either as we have done in processing the RTS or in providing scheduling for multiple processes or threads.

The EMP [11] project at Ohio Supercomputer Center takes offloading one step further and provides matching for MPI messages on the NIC. Using similar hardware (Alteon Acenic NICs and relatively slow Pentium processors), they report impressive performance numbers: 880 $\mathrm{Mb} / \mathrm{s}$ bandwidth and $23 \mu$ s latency. In contrast to the EMP approach, we have started from the perspective that the OS should manage the resources provided by the NIC. This includes buffer space, information about messages in transit, and flow control. EMP starts all resource management on the NIC or in the application. We are currently in the process of migrating more of this management into the NIC and the EMP project is looking into providing 
some NIC management in the OS.

\section{Conclusions and Future Work}

Through our experiments, we have shown that protocol offloading is a viable approach to reducing the processor capacity bottleneck and improving communication performance. So far, we have only offloaded the handling associated with data packets. All control packets (with the exception of CTS packets) are handled by the OS and, in particular, the RMPP and Portals modules. When we started this work, we expected to offload all of RMPP and Portals to the NIC. And, while we plan to continue selectively offloading more of the protocol processing, this represents an interesting milestone in our efforts.

While programmable NICs are a great asset in the kinds of studies that we are engaged in, they are never pricecompetitive with NICs that do not support a programming interface. Because the processing that we have offloaded is very straightforward and the resource management is done by the OS, one could easily imagine the development of NICs that incorporate only this level of processing. In this context, it is worth noting that the Portals module simply provides an answer to the question of where an incoming message should be placed. While we have focused on MPI-based message passing, it is interesting to consider other ways that this question might be answered. One obvious possibility is to have the OS match the incoming data with the application buffer provided in a socket read operation. Additionally increasing the MTU to a reasonable size would provide a simple, true zerocopy TCP capability that has been so elusive [5].

We intend to examine other parts of the protocol processing that could be offloaded to the NIC. While it might be natural to assume that we would next offload the retransmit logic to the NIC (as is done in EMP), we plan to leave this in part of the protocol processing in the OS. First, because we expect that dropped packets will be infrequent, we would prefer to keep this part of the protocol processing out of the fast path whenever as possible. Second, this part of the protocol processing is most closely related to resource management and naturally belongs in the OS.

Rather than offloading the retransmission logic, we will explore ways in which the matching of incoming mes- sages to pre-posted receives can be offloaded to the NIC. The goal is to identify a general purpose matching strategy that could be used for a variety of upper level protocols and implement this on the NIC. If we can provide the NIC with enough information to match an incoming message with a pre-posted receive, the NIC will be able to: 1) notify the local OS that it has initiated a receive, 2) generate and send a CTS to enable the flow of more data packets, and 3) transfer the initial and subsequent data packets directly to the application. This should result in a significant reduction in latency for small messages.

\section{Acknowledgements}

This work would not have been possible without support from a variety of places. Much of the UNM effort was funded by a contract from Sandia National Laboratories. The Alteon Acenic NICs were borrowed from the Albuquerque High Performance Computing Center and were acquired as part of an IBM SUR (Sponsored University Research) grant.

We also had a great deal of support from our colleagues in the Scalable Systems Lab at UNM. Patricia Gilfeather and Todd Underwood were very helpful in our initial efforts to get code running on the Acenics. Bill Lawry and Riley Wilson provided the benchmarking tool used to obtain the processor availability results.

Finally, Pete Wyckoff from the Ohio Supercomputer Center was very helpful in providing debugging support and guidance in subtle programming issues related to the Acenics. Pete also read an early draft of this paper and offered many helpful suggestions

\section{References}

[1] Ron Brightwell, Tramm Hudson, Rolf Riesen, and Arthur B. Maccabe. The portals 3.0 message passing interface. Technical Report SAND99-2959, Sandia National Laboratories, December 1999.

[2] Compaq, Microsoft, and Intel. Virtual interface architecture specification version 1.0. Technical report, Compaq, Microsoft, and Intel, December 1997. 
[3] Patricia Gilfeather and Todd Underwood. Fragmentation and high performance IP. In CAC Workshop, April 2001.

[4] Rick Jones. The network performance home page. http://www.netperf.org/netperf/NetperfPage.html.

[5] Christian Kurmann, Michel Müller, Felix Rauch, and Thomas M. Stricker. Speculative defragmentation-a technique to improve the communication in software efficiency for gigabit ethernet. In Proceedings of the 9th International Symposium on High Performance Distributed Computing (HPDC), August 2000.

[6] Mario Lauria, Scott Pakin, and Andrew Chien. Efficient layering for high speed communication: Fast messages 2.x. In Proceedings of the IEEE International Symposium on High Performance Distributed Computing, 1998.

[7] Arthur B. Maccabe, William Lawry, Christopher Wilson, and Rolf Riesen. Distributing application and OS functionality to improve application performance. Technical Report TR-CS-2002-11, Computer Science Department, The University of New Mexico, April 2002.

[8] Message Passing Interface Forum. MPI: A messagepassing interface standard. The International Journal of Supercomputer Applications and High Performance Computing, 8, 1994.

[9] Myricom, Inc. The GM message passing system. Technical report, Myricom, Inc., 1997.

[10] Rolf Riesen. Message-Based, Error-Correcting Protocols for Scalable High-Performance Networks. $\mathrm{PhD}$ thesis, The University of New Mexico, Computer Science Department, Albuquerque, NM 87131, 2002.

[11] Piyush Shivam, Pete Wyckoff, and Dhabaleswar Panda. EMP: Zero-copy OS-bypass NIC-driven gigabit Ethernet message passing. In Supercomputing, November 2001.
[12] Task Group of Technical Committee T11. Information technology - scheduled transfer protocol - working draft 2.0. Technical report, Accredited Standards Committee NCITS, July 1998.

[13] Todd Underwood. Fragmentation as a strategy for high-speed IP networking. Master's thesis, The University of New Mexico, Computer Science Department, Albuquerque, NM 87131, 2001.

[14] Wenbin Zhu. OS bypass investigation and experimentation. Master's thesis, The University of New Mexico, Computer Science Department, Albuquerque, NM 87131, 2002. 